\title{
Bowen's disease with multiple locations: Clinical presentation and therapeutic approach
}

\author{
Jean-Baptiste Andonaba', Nina Korsaga/Some², Boukary Diallo', Issouf Konate", \\ Valentin Konsegre ${ }^{3}$
}

${ }^{1}$ Department of Dermatology and Venereology, Sourô Sanou University Teaching Hospital of Bobo-Dioulasso (CHUSS), Boni Nazi University of Bobo-Dioulasso, Higher Institute of Health Sciences (INSSA), Burkina Faso, ${ }^{2}$ Department of Dermatology and Venereology Yalgado Ouédraogo University Teaching Hospital of Ouagadougou (CHUSS), Joseph Ki-Zerbo University of Ouagadougou, UFR/SDS Health Sciences (INSSA), Burkina Faso, ${ }^{3}$ Department of Pathological Anatomy, Sourô Sanou University Teaching Hospital of Bobo-Dioulasso (CHUSS), Boni Nazi of Bobo-Dioulasso (UPB), Higher Institute of Health Sciences (INSSA), Burkina Faso

Corresponding author: Prof. Jean-Baptiste Andonaba, E-mail: jb_andonaba@yahoo.fr

\begin{abstract}
The Bowen's disease (MB), also known as squamous cell carcinoma in situ is a neoplastic skin disease that was clinically and histologically individualized by Darier in 1914. The isolated lesions in the majority of patients can be multiplied in 10 to $20 \%$ of cases. We report many locations which has posed problems of therapeutic choice. It is the case of a 35-year-old housewife, seen in consultation for red colored and squamous patches associated to pruritus and pain. On the skin, the examination found red colored and squamous patches, in projection, shaped rounded with a very slow evolution combining in a variable manner, rashes, scales, crusts and keratosis. Patches tended to grow progressively. The vulvar lesions were pigmented patches. In the mouth, we noted ulcers and a diffuse hypopigmentation with cheilitis, perlèche and odynophagia. The oncology checkup in search of another skin, mucosal and visceral cancer was done without any cancer found and the general condition was retained. The histopathology performed on two biopsies confirmed the diagnosis. Considering the spread of lesions and after a pretreatment evaluation, we put the patient under 5 fluorouracil in cream on evening and under isotretinoin capsule with favorable evolution in the third week. The multiple sites of $\mathrm{MB}$ are rare but pose a management problem because of the evolutionary potential of each individual lesion in squamous cell carcinoma. The choice of treatment depends on the general condition of the patient, the expected efficacy of the treatment but also on its tolerance and cost.
\end{abstract}

Key words: Bowen's disease; Multiple locations; Symptoms; Treatment; Burkina faso

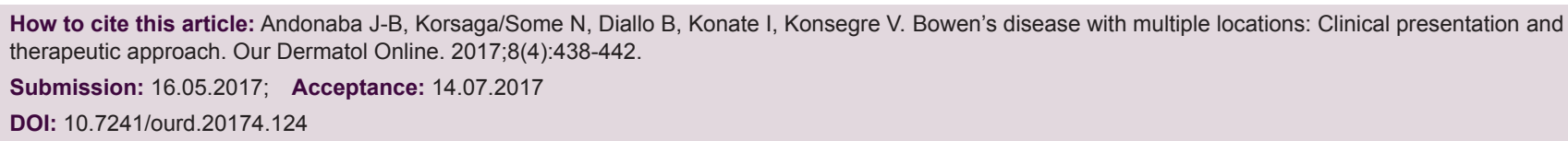




\title{
Maladie de Bowen à localisations multiples: présentation clinique et approche thérapeutique
}

\author{
Jean-Baptiste Andonaba', Nina Korsaga/Some², Boukary Diallo', Issouf Konate', \\ Valentin Konsegre ${ }^{3}$
}

${ }^{1}$ Department of Dermatology and Venereology, Sourô Sanou University Teaching Hospital of Bobo-Dioulasso (CHUSS), Boni Nazi University of Bobo-Dioulasso, Higher Institute of Health Sciences (INSSA), Burkina Faso, ${ }^{2}$ Department of Dermatology and Venereology Yalgado Ouédraogo University Teaching Hospital of Ouagadougou (CHUSS), Joseph Ki-Zerbo University of Ouagadougou, UFR/SDS Health Sciences (INSSA), Burkina Faso, ${ }^{3}$ Department of Pathological Anatomy, Sourô Sanou University Teaching Hospital of Bobo-Dioulasso (CHUSS), Boni Nazi of Bobo-Dioulasso (UPB), Higher Institute of Health Sciences (INSSA), Burkina Faso

Corresponding author: Prof. Jean-Baptiste Andonaba, E-mail: jb_andonaba@yahoo.fr

\begin{abstract}
RESUMÉ
La maladie de Bowen (MB) est un carcinome in situ malpighien intra-épithélial relativement rare qui fut individualisée sur le plan clinico-histologique par Darier en 1914. Les lésions isolées chez la majorité des patients peuvent être multiples dans 10 à 20\% des cas. Nous rapportons un cas à localisations multiples qui a posé des problèmes de choix thérapeutique. Il s'est agi d'une ménagère de 35 ans, vue en consultation pour lésions érythématosquameuses prurigineuses et douloureuses. Sur la peau, on retrouvait des plaques érythématosquameuses à peine saillante, discoïde avec une évolution très lente associant, de façon variable, un érythème, des squames croûtes et une kératose. Les lésions vulvaires pigmentées étaient en relief sous forme de plaques. Au niveau buccal on notait les lésions aphtoïdes et une leucoplasie diffuse avec chéilite, perlèche et odynophagie. Le bilan carcinologique à la recherche d'un cancer cutané, muqueux ou viscéral était sans particularité notable et l'état général était conservé. L'histopathologie réalisée sur deux biopsies a confirmé le diagnostic. Devant l'étendue des lésions et après un bilan préthérapeutique nous avons mis la patiente sous 5FU en crème le soir et sous isotrétinoïne gélule avec évolution favorable à la troisième semaine. Les localisations multiples de la MB sont rares mais posent un problème de prise en charge, compte tenu du potentiel évolutif de chaque lésion prise individuellement en carcinome épidermoïde. Le choix du traitement dépend de l'état général du patient, de l'efficacité attendue du traitement mais aussi de sa tolérance et son coût.
\end{abstract}

Key words: Bowen; Lésions multiples; Clinique; Traitement; Burkina faso

\section{INTRODUCTION}

La maladie de Bowen (MB) est un carcinome in situ malpighien intra-épithélial relativement rare. Décrite par John T. Bowen en 1912 qui avait déjà reconnu sa nature précancéreuse, elle fut ensuite individualisée sur le plan clinico-histologique par Darier en 1914. La $\mathrm{MB}$ atteint surtout l'adulte à tout âge. La courbe d'âge s'étale de 18 à 95 ans, avec une médiane à 65 ans. Les lésions peuvent être isolées ou multiples (10 à $20 \%$ des cas) [1]. Elle se présente sous forme de plaque persistante, progressive, plate, rouge, squameuse ou croûteuse induite par un carcinome intradermique et potentiellement maligne. Des cellules atypiques de l'épithélium malpighien prolifèrent dans la profondeur de l'épiderme. Les lésions peuvent se produire sur toute la surface de la peau ou sur les muqueuses. La MB peut progresser dans 3 à 5 p. 100 des cas en carcinome invasif, dans des délais très variables [2]. Elle s'associe volontiers à d'autres carcinomes cutanés (baso- ou spinocellulaires) [3]. Son caractère paranéoplasique comme marqueur de cancer viscéral associé est actuellement réfuté [4]. Les facteurs favorisants de maladie de Bowen sont: la lumière solaire, l'arsenic,

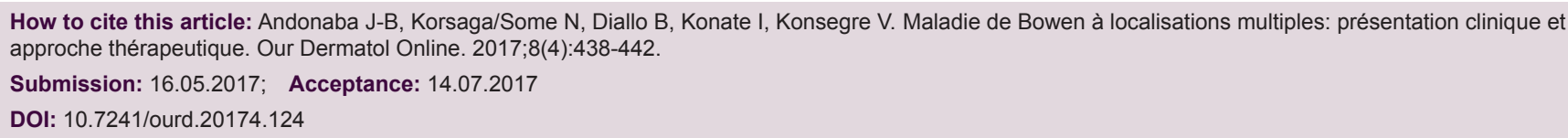


l'immunosuppression, l'infection HPV [5]. Elle peut s'associer avec ou compliquer certaines dermatoses chroniques: lupus vulgaire, lupus érythémateux chronique. Nous rapportons un cas à localisations multiples posant le problème des indications des différents moyens thérapeutiques disponibles.

\section{CASE REPORT}

SN, 35 ans, ménagère a été vue en consultation pour lésions érythématosquameuses prurigineuses et douloureuses. Lévolution remonterait à trois mois marquée par la survenue de macules érythémateuses sur la région ombilicale qui se sont étendues secondairement sur le tronc, la région vulvaire et les quatre membres. Sur la peau, on retrouvait des plaques érythématosquameuses à peine saillante, discoïde avec une évolution très lente associant, de façon variable, un érythème, des squames croûtes et une kératose (Fig. la). À la palpation, les lésions étaient légèrement infiltrée, douloureuses avec signe de Nikolsky présent. Le prurit entraînait des exulcérations qui cicatrisaient pour laisser des cicatrices squameuses et pigmentées. Par endroits, les lésions de la peau prenaient un aspect annulaire et une évolution centrifuge. Les lésions vulvaires pigmentées étaient en relief sous forme de plaques (Fig. 2a). Au niveau buccal on notait les lésions aphtoïdes et une leucoplasie diffuse avec chéilite, perlèche et odynophagie. L'examen au spéculum n'a pas retrouvé une localisation de la maladie. L'histologie a montré un revêtement épidermique hyperkératosique parakératosique acanthosique, papillomateux et reposant sur une basale régulière. On observait au niveau du corps muqueux de Malpighi des cellules basophiles à noyaux élargis, hyperchromatiques et à nucléoles proéminents ainsi que des foyers de maturation kératosique (Fig. 3). Le bilan carcinologique à la recherche d'un cancer cutané, muqueux ou viscéral était sans particularité notable et l'état général était conservé. Devant l'étendue des lésions et après un bilan préthérapeutique nous avons mis la patiente sous $5 \mathrm{FU}$ en crème le soir et sous isotrétinoïne gélule (20 mg par jour). Lévolution a été favorable à la troisième semaine avec disparition de nombreuses lésions, blanchiments d'autres (Figs. $1 \mathrm{~b}$ et $2 \mathrm{~b}$ ) et absence de nouvelles lésions et de signes fonctionnelles. Une surveillance mensuelle sous traitement a été instituée. Le pronostic chez notre patiente est bon au vu de la réponse thérapeutique sous cette bithérapie et l'absence d'un cancer associé.

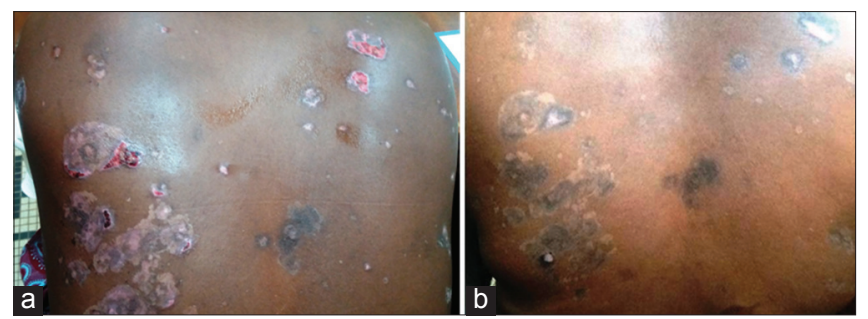

Figure 1: Lésions érythématosquameuses exulcérées par endroits, à contours polycycliques (1a). Evolution locale assez favorable après 3 semaines de traitement (1b).
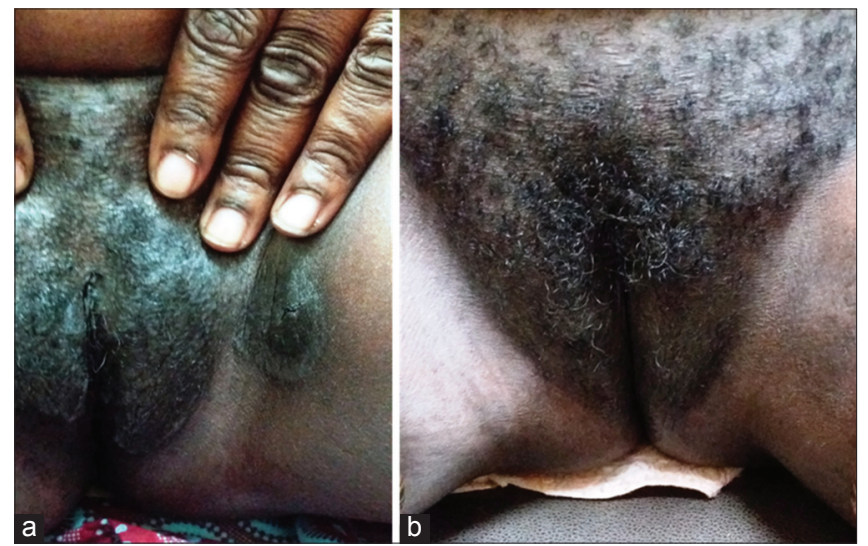

Figure 2: Lésions annulaires squameuses et hyperpigmentées sur l'aisne et la face externe des grandes lèvres (2a). Noter le nettoyage des lésions après 3 semaines de traitement $(\mathbf{2 b})$.

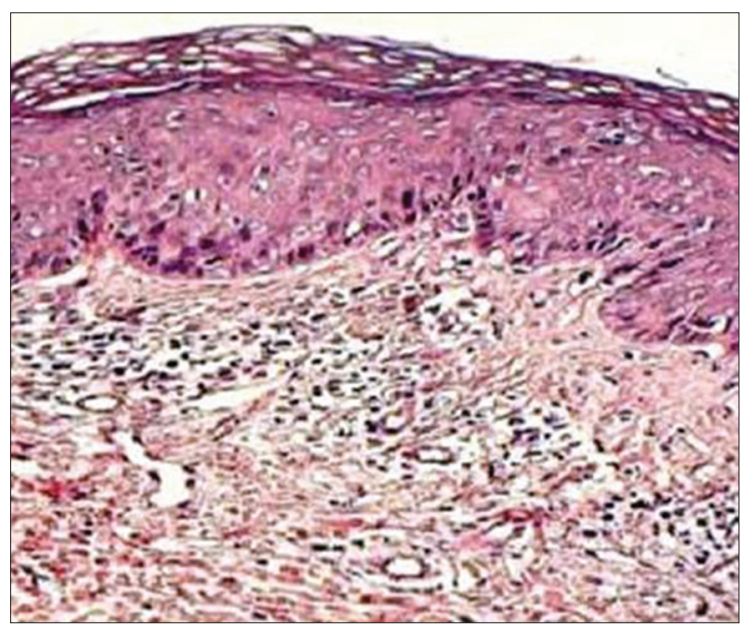

Figure 3: Images histopathologiques montrant une anarchie cellulaire et des atypies qui restent cantonnées à l'épiderme avec une basale épidermique respectée. On note un infiltrat inflammatoire du chorion.

\section{DISCUSSION}

Les formes multiples sont observées dans 10 à $20 \%$ des cas et les femmes sont les plus touchées [6]. La courbe d'âge s'étale de 18 à 95 ans, avec une médiane à 65 ans [1]. Notre cas remplissait tous les critères d'âge et de sexe. La MB atteint préférentiellement les zones photo exposées mais peut toucher n’importe 
quelle zone de la peau, couverte ou non couverte et les muqueuses, surtout génitales. Les formes génitales touchent plus souvent l'adulte jeune avec une prédominance féminine [6]. Son diagnostic clinique peut être délicat surtout la forme génitale pigmentée observée dans notre cas et décrit par Bounouar et al. [7]. Cette difficulté diagnostique est accentuée par le fait que plusieurs maladies peuvent simuler une MB. Les diagnostics différentiels comprennent: le psoriasis, le lupus érythémateux, la verrue séborrhéique, la kératose actinique, le lichen verruqueux ou encore le carcinome basocellulaire superficiel. Lexamen histopatologique est la clé du diagnostic de MB; elle montre des images habituellement caractéristiques. On note d'emblée une altération de la cellule malpighienne et une perte de l'architecture épithéliale normale cantonnée à l'épiderme. Cette anarchie cellulaire et les atypies restent cantonnées à l'épiderme et la basale épidermique est respectée comme dans notre résultat anatomopathologique. Il faut d'ailleurs s'assurer par des coupes sériées, surtout s'il existe une MB génitale, qu'il n'existe pas de rupture de la basale en aucun point du prélèvement. La maladie de Bowen peut progresser dans 3 à 5 p. 100 des cas en carcinome invasif, dans des délais très variables [2]. Elle s'associe volontiers à d'autres carcinomes cutanés (baso ou spinocellulaires) [3]. Ce qui justifie un bilan carcinologique qui peut revenir négatif comme dans notre cas (facteur de bon pronostic). Le traitement de choix reste l'exérèse chirurgicale précoce et complète, confirmée par l'examen histologique de la ou des lesions de $\mathrm{MB}$ compte tenu de son potentiel évolutif en carcinome spinocellulaire. La fréquence des récidives est environ de $5 \%$ pour les MB cutanées et de $10 \%$ pour les localisations vulvaires si la marge d'exerce passe en peau saine. Au contraire, lorsque cette marge n'est pas atteinte, les récidives atteignent $50 \%$ dans les $\mathrm{MB}$ vulvaires. Cependant pour les localisations multiples comme dans notre observation, d'autres méthodes ont été proposées pour la destruction complète et précoce de la lésion de maladie de Bowen et le choix est fonction du type de lésion, du siège, de son évolution, de l'extension et du terrain. Il s'agit des agents antimitotiques locaux, de la radiothérapie, de la cryothérapie, du curetage-électrodessiccation, du laser, de la thérapie photodynamique et autres moyens thérapeutiques. Létrétinate, l'isotrétinoïne et les interférons $\alpha$ et $\gamma$ ont été essayés en cas de lésions multiples et surtout vulvaires associées à des lésions cervicales dysplasiques, sans que leur efficacité soit suffisante [8]. Dans la littérature, il n'existe actuellement pas d'essais cliniques contrôlés, randomisés entre notamment l'approche chirurgicale, les traitements topiques et la Mal-PDT (utilisation de l'aminolevulinate de méthyle ou MAL comme sensibilisateur associé à une thérapie photodynamique). Ceci est rappelé dans la métaanalyse publiée en 2013 et qui souligne que très peu de données méthodologiquement correctes sont publiées pour les approches chirurgicales et traitements topiques de la MB [9]. Concernant la Mal-PDT, en terme de clearance tumorale, elle parait significativement plus efficace que la cryothérapie, en revanche, il n'y a pas de différence statistiquement significative quand la PDT est comparée aux applications locales de 5-FU. De même cette méta analyse ne retrouve pas de différence statistiquement significative quand la cryothérapie est comparée aux applications locales de 5-FU. Nous avons choisi d'associer des applications locales de 5-FU + isotrétinoïne orale avec de bons résultats à la troisième semaine.

\section{CONCLUSION}

Les localisations multiples cutanéomuqueuses de la MB sont rares mais posent un problème de prise en charge, compte tenu du potentiel évolutif de chaque lésion prise individuellement en carcinome épidermoïde. Le choix du traitement dépend d'un certain nombre d'éléments comme: l'état général du patient, l'accès du patient et du praticien à tel ou tel type de traitement, l'observance du patient, l'efficacité attendue du traitement mais aussi sa tolérance et son coût. Lassociation 5-FU + isotrétinoïne s'est révélée à court terme efficace dans chez notre patiente avec des localisations multiples. Quelle que soit la méthode choisie, une surveillance régulière à vie du patient s'impose, afin de dépister précocement les récidives ou l'apparition d'une autre tumeur cutanée maligne non mélanocytaire notamment. Des travaux récents ont montré que la MB n'est pas un marqueur de cancer solide. Il n'est donc pas justifié de pratiquer des bilans répétés, traumatisants et coûteux, à la recherche d'un cancer viscéral chez les patients traités pour une MB.

\section{REFERENCES}

1. Lee MM, Wick MM. Bowen's disease. Cancer J Clin, 1990, 40: 237242.

2. Kao GF. Carcinoma arising in Bowen's disease. Arch Dermatol, 1986, 122: 1124-1126.

3. Reizner GT, Chuang TY, Elpern DJ et al. Bowen's disease in Kauai, Hawaii. A population-based incidence report. J Am Acad Dermatol, 1994, 31: 596-600.

4. Chute CG, Chuang TY, Bergstrahl EJ, Su WP. The subsequent risk 


\section{www.odermatol.com}

of internal cancer with Bowen's disease. A population based study. JAMA, 1991, 266: 916-919.

5. Mcgregor JM, Proby CM. The role of papillomaviruses in human non melanoma skin cancer. Cancer Surv, 1996, 26: 219-236.

6. Kovacs A, Ynemoto K, Katsuoka K et al. Bowen's disease: statistical study of a 10 year period. J Dermatol, 1996, 23: 267-274.

7. Mariem Bounouar, Fatimazahra Mernissi. Maladie de Bowen pigmentée génitale. Pan African Medical Journal. 2014; 19:192 doi:10.11604/pamj.2014.19.192.2629

8. Gordon KB, Roenick HH, Gendleman M.Treatment of multiple lesions of Bowen disease with isotretinoin and interferon alpha.
Arch Dermatol, 1997, 133: 691-693.

9. Bath-Hextall FJ, Matin RN, Wilkinson D, Leonardi-Bee J. Interventions for cutaneous Bowen's disease. Cochrane database Syst Rev, 2013, 24: 6: CD 007281

Copyright by Jean-Baptiste Andonaba, et al. This is an open-access article distributed under the terms of the Creative Commons Attribution License, which permits unrestricted use, distribution, and reproduction in any medium, provided the original author and source are credited.

Source of Support: Nil, Conflict of Interest: None declared. 